\title{
Insecticides Susceptibility Status of Malaria Vectors in a High Malaria Endemic Tribal District Gadchiroli (Maharashtra) of India
}

\author{
$\underline{\text { RK Singh', Gaurav Kumar }}{ }^{2}$, Kunal S Modak ${ }^{3}$, Rajesh R Karlekar $^{4}$, $\underline{\text { RC Dhiman }}^{5}$ \\ 1,2,5ICMR-National Institute of Malaria Research, Sector-8, Dwarka, New Delhi, India. \\ ${ }^{3} \mathrm{DMO}$, Gadchiroli, Maharashtra, India. \\ ${ }^{4}$ NVBDCP Consultant, DMO Office, Gadchiroli, Maharashtra, India.
}

DOI: https://doi.org/10.24321/0019.5138.202137

\section{I $\quad \mathbf{N} \quad \mathbf{F} \quad \mathbf{O}$}

\section{Corresponding Author:}

RK Singh, ICMR-National Institute of Malaria Research, Sector-8, Dwarka, New Delhi, India.

E-mail Id:

singhriku@yahoo.co.in

Orcid Id:

https://orcid.org/0000-0002-5909-9725

How to cite this article:

Singh RK, Kumar G, Modak KS, Karlekar RR, Dhiman RC. Insecticides Susceptibility Status of Malaria Vectors in a High Malaria Endemic Tribal District Gadchiroli (Maharashtra) of India. J Commun Dis. 2021;53(3):33-50.

Date of Submission: 2021-04-01

Date of Acceptance: 2021-07-05

\section{$\begin{array}{llllllll}\mathbf{A} & \mathbf{B} & \mathbf{S} & \mathbf{T} & \mathbf{R} & \mathbf{A} & \mathbf{C} & \mathbf{T}\end{array}$}

Background and Objective: The current study was undertaken to determine insecticide susceptibility of malaria vectors in various villages of high malaria endemic PHCs of Gadchiroli district of Maharashtra.

Methods: Adult malaria vectors were collected from the human dwellings/ cattle sheds of 156 villages of 18 malaria endemic PHCs. Susceptibility tests were carried out for different insecticides against An. culicifacies and An. fluviatilis mosquitoes as per the World Health Organization (WHO) procedure. Cone bioassays were also done to assess the quality and efficacy of indoor residual spray.

Results: An. fluviatilis could be collected from 23 villages only and all the populations were fully susceptible to synthetic pyrethroid (deltamethrin) while being tolerant to organophosphorous (malathion). Susceptibility of An. culicifacies from 156 villages indicated that only 3 populations of $A n$. culicifacies were resistant to deltamethrin while 57 populations were fully susceptible and other 96 populations were tolerant to deltamethrin. Resistance was recorded in 25 populations of An. culicifacies against malathion and 30 populations were tolerant to malathion insecticide. Remaining populations of An. fluviatilis and An. culicifacies were highly resistant to organochlorine. Results of cone bioassay revealed the mortality ranged from $32.5-51.1 \%$ on cemented and $27.5-43.3 \%$ on the mud wall sprayed with lambda cyhalothrin.

Conclusion: The current study indicates that resistance has developed to synthetic pyrethroids in the major malaria vector An. culicifacies. Therefore, there is an urgent need for the evaluation of new insecticide molecules for better control of malaria vectors.

Keywords: Malaria Vector, Insecticide Susceptibility, Gadchiroli District of Maharashtra, India 


\section{Introduction}

Malaria is the most common vector-borne disease and it is curable if effective treatment is started early. Delay in the treatment of malaria may cause serious health problems and may even lead to death. Many people are being affected by the disease in tropical and sub-tropical countries. 228 million people are at the risk of being infected with malaria and nearly four lac people died in 2018 from this disease globally. ${ }^{1}$ Malaria is one of the oldest and highly endemic diseases throughout India, and it is a major cause of mortality and morbidity among human population with 429928 cases and 96 deaths reported during the year $2018 .^{2}$ The central part of the country is a high malaria endemic region where about $90 \%$ of the population is at risk of infection of malaria. ${ }^{3}$ In Maharashtra, 10757 malaria cases and 13 deaths were reported in 2018 by National Vector Borne Disease Control Programme (NVBDCP), ${ }^{2}$ and Vector Borne Disease Control Programme of the state was benefited from the enhanced malaria control project (EMCP) funded by the World Bank since 1997. ${ }^{4}$

Gadchiroli district of Maharashtra is a tribal district in the central part of the country and one of the high malaria endemic districts, where 34206 malaria positive cases were reported in the year 2015, out of which Plasmodium falciparum (PF) accounted for $79.6 \%$. Slide positivity rate (SPR) in Gadchiroli has been ranging from as low as $2.57 \%$ to as high as $4.57 \%$ between the years 2011 and 2015. ${ }^{5}$

Malaria disease is caused by a protozoan parasite of genus Plasmodium in humans. Four species of malaria parasites of genus Plasmodium, namely Plasmodium vivax, P. falciparum, $P$. malariae and $P$. ovale are responsible for the disease, of which $P$. vivax and $P$. falciiparum are both the common malarial parasites responsible for global mortality including in India. ${ }^{6-9}$ In India, malaria is transmitted by bites of female Anopheles mosquitoes of An. culicifacies, An. stephensi, An. fluviatilis, An. minimus, An. dirus, and An. sundaicus, the six primary vectors, and An. varuna, An. annularis, An. philippinensis and An. jeyporiensis the four secondary vectors of malaria. An. culicifacies alone contributes more than $65 \%$ of the total cases of malaria annually and is found widely in rural and peri-urban areas. ${ }^{8}$ An. culicifacies and An. fluviatilis are the main malaria vectors in India. An. culicifacies is the common primary malaria vector found throughout the year while An. fluviatilis is found mostly in the winter season.

The use of insecticides is an important tool for reducing the density of adult Anopheles malaria vector for malaria control. Vector control is a major part of the strategy of Vector Borne Disease Control Programme and two types of vector control methods, indoor residual spray (IRS) and insecticide-treated bed nets (ITBNs)/ long-lasting insecticide treated nets (LLINs) are used to control malaria transmission. ${ }^{6-8}$ In spite of these control measures, malaria remains a major public health problem in India. IRS with synthetic insecticides like DDT and BHC was used to control malaria vectors under malaria control programme in India since 1958, but due to continued use of synthetic insecticides, An. culicifacies has developed resistance against DDT, dieldrin, and malathion in few districts of Maharashtra such as Gadchiroli, and in Gujarat. ${ }^{10-11}$ Synthetic pyrethroid (deltamethrin) was introduced in IRS in 1998 in Gadchiroli district. 5,12,13 Later in 2009, other synthetic pyrethroids as alphcypermethrin, cyfluthrin and lambda cyhalothrin were used for malaria control and ITBNs/ LLINs were distributed in Gadchiroli district. Synthetic pyrethroids are used in IRS, impregnation of bed nets and making LLINs to control malaria. More than fifteen years ago, the resistance in An. culicifacies against synthetic pyrethroids (deltamethrin) was reported from Surat district of Gujarat ${ }^{14}$ and malaria vectors also reported tolerant/ partial resistance to synthetic pyrethroids used in IRS. However, malaria vectors have developed resistance to DDT in 286 districts, to malathion in 81 and to pyrethroids in 2 districts in India due to selection pressure. ${ }^{6-8}$ Presently, Gadchiroli district has been receiving two rounds of IRS with synthetic pyrethroids and regular use of ITBNs/ LLINs every year. ${ }^{5,12,13}$

In order to find out the current status of insecticide resistance in malaria vectors of Gadchiroli, the current study was undertaken. An in-depth monitoring of insecticide susceptibility of An. culicifacies and An. fluviatilis malaria vectors against various insecticides like lambda cyhalothrin, permethrin, cyfluthrin, deltamethrin, malathion and DDT was done in 156 endemic villages of various PHCs of Gadchiroli district. This information may be very useful to finalise the strategy for malaria prevention and elimination of malaria from Gadchiroli district of Maharashtra state.

\section{Materials and Methods}

\section{Study Site}

The Gadchiroli district of Maharashtra was created in 1982 from Chandrapur district. It is geographically a large district with a geographical area of $14412 \mathrm{~km} .{ }^{2}$ It is situated at $18.43^{\prime}$ to $21.50^{\prime} \mathrm{N}$ latitude and $79.45^{\prime}$ to $80.53^{\prime} \mathrm{E}$ longitude and has uneven terrain with hills, valleys, and forests at different altitudes. It has a total population of 1072942 as of census $2011 .{ }^{15}$ The average rainfall is $1743.5 \mathrm{~mm}$, minimum temperature $11.3^{\circ} \mathrm{C}$ and maximum $47.7^{\circ} \mathrm{C}$. Gadchiroli is a tribal dominant district and most of the people are Gond tribals. Their houses have mud-plastered walls with thatched/tiled roofs and all the cattle sheds are near human dwellings. These types of houses are more suitable for malaria vectors to rest and bite the host, thereby spreading malaria rapidly. In tribal and forest areas, malaria control is a very difficult task due to various topographical, socioeconomical and ecological factors. Water reservoirs with 
streams, drains and springs and water lodged in the downhill are the main sources of immature stages of mosquitoes.

\section{Analysis of Malaria Epidemiological Data}

The Primary Health Centre $(\mathrm{PHC})$ wise malaria epidemiological data were collected for five years, i.e. from 2011-15, from the District Malaria Officer (DMO), Gadchiroli district of Maharashtra state. The data were also analysed to obtain various parameters like annual blood examination rate (ABER), slide positivity rate (SPR), slide falciparum rate (SFR), Pf percent and number of deaths (Table 1 ).

Table I. Epidemiological Data of Malaria in Gadchiroli District of Maharashtra (20 I I-20 I5)

\begin{tabular}{|c|c|c|c|c|c|c|c|c|c|c|c|}
\hline Year & Population & BSC\&E & +ve & PF & PV & ABER & API & SPR & SFR (\%) & PF (\%) & Death \\
\hline 2011 & 1158947 & 529846 & 13670 & 10581 & 3089 & 45.72 & 11.80 & 2.57 & 1.99 & 77.40 & 6 \\
\hline 2012 & 1177242 & 520258 & 6596 & 4656 & 1940 & 44.19 & 5.60 & 1.26 & 0.89 & 70.58 & 4 \\
\hline 2013 & 1209496 & 536341 & 6436 & 4919 & 1517 & 44.34 & 5.32 & 1.19 & 0.91 & 76.42 & 6 \\
\hline 2014 & 1187784 & 692277 & 24469 & 21410 & 3059 & 57.23 & 20.23 & 3.53 & 3.09 & 87.49 & 18 \\
\hline 2015 & 1204101 & 747113 & 42062 & 7228 & 6978 & 62.7 & 28.40 & 4.57 & 3.64 & 79.60 & 11 \\
\hline
\end{tabular}

Table 2.List of Villages and PHCs in Gadchiroli (Maharashtra) selected for Insecticide Susceptibility Study undertaken on Malaria Vectors

\begin{tabular}{|c|c|c|}
\hline Name of PHCs & Name of Sub Centres & Name of Study Villages \\
\hline \multirow{3}{*}{ Kotgul } & Sonpur & Sonpur, Bhimankozi \\
\hline & Gyarahpatti & Gyarahpatti, Pitesur gaon \& tola, Nehalgad \\
\hline & Kosami 2 & Kosami 2 \\
\hline \multirow{3}{*}{ Malewada } & Devsara & Devsara, Mendha, Murmadi, Khatitola \\
\hline & Khobramenda & Khobramenda, Huryal dand, Badbada, Yeduskuhi \\
\hline & Jaysing Tola & Wagha bhumi, Mange wada, Jaysing tola, Khamtala \\
\hline \multirow{3}{*}{ Gatta } & Jhanbia & Jhanbia gaon \& tola, Andenge, Nander \\
\hline & Hedari & Hedari gaon, Mallam pahari, Surjagad \\
\hline & Gatta & Gatta gaon \& tola, Mohndi gaon, Tadguda \\
\hline \multirow{5}{*}{ Kasansur } & Kasansur & Venasur, Chokhe wada, Jhuri \\
\hline & Kotami & Kotami \\
\hline & Kondawahi & Etawahi, Kondawahi \\
\hline & Tadguda & Tadguda \\
\hline & Ghotsur & Ghotsur, Jaweli \\
\hline \multirow{5}{*}{ Todsa } & Ekrakhurd & Ekrakhurd, Ekrabhurj \\
\hline & Aalinga & Aalinga gaon \& tola, Zarewada, Alandi \\
\hline & Tadhpalli & Tadhpalli, Lanji, Karampalli gaon \& tola \\
\hline & Todsa & Petha gaon \\
\hline & Devada & Devada \\
\hline \multirow{4}{*}{ Aarewada } & Kiyar & Hemal kasha, Karampalli gaon \& tola \\
\hline & Aarewada & Aarewada, Medpalli \\
\hline & Bhamaragad & Dubba guda \\
\hline & Tadgaon & Tadgaon, Dhulepalli, Erakdumme \\
\hline \multirow{4}{*}{ Laheri } & Dhondraj & Dhondraj, Ranipudar \\
\hline & Laheri & Laheri, Murangal, Aaldandi, Gundenoor \\
\hline & Malum padur & Kuka menda, Malum padur, Bhoose wada \\
\hline & Beena gunda & Beena gunda \\
\hline
\end{tabular}




\begin{tabular}{|c|c|c|}
\hline \multirow{3}{*}{ Manne Rajaram } & Manne Rajaram & Mokela \\
\hline & Chichoda & Chichoda, Jijgaon, Bhade nagar \\
\hline & Yechali & Yechali, Basa guda, Rela, Rai guda, Maram palli, Lakan duga \\
\hline \multirow{3}{*}{ Godalwahi } & Godalwahi & Godalwahi gaon, Godalwahi tola, Michgaon \\
\hline & Sallebhatti & Sallebhatti, Lekha, Kannartola, Mendha \\
\hline & Girola & Udegaon \\
\hline \multirow{5}{*}{ Pendhari } & Pendhari & Pulkal, Dhorgatta, Pekinmudza \\
\hline & Durgapur & Durgapur, Khargi \\
\hline & Chichoda & Chichoda, Sinsur \\
\hline & Kaneli & Kaneli, Paidi \\
\hline & Gatta & Gota gaon \& tola, Jhawal zora, Pather gatta \\
\hline \multirow{7}{*}{ Karwafa } & Kondawahi & Kondawahi gaon \& tola, Fulbodi, Zari \\
\hline & Fulbodi & Kanartola \\
\hline & Pustola & Pustola, Kopagoda \\
\hline & Karwafa & Talodhi, Navegaon \\
\hline & Sakhera & Sakhera, Jamblitola \\
\hline & Chatgaon & Katezari \\
\hline & Mendhatola & Mendhatola, Khutgaon, Michgaon \\
\hline \multirow{8}{*}{ Murumgaon } & Savargaon & $\begin{array}{l}\text { Morchul, Savar gaon, Markagaon \& tola, Gajamendi, Kangadi, } \\
\text { Makdand }\end{array}$ \\
\hline & Khambada & Tadegaon, Dongarhud \\
\hline & Sursundi & Sursundi, Eurup dodari, Saygaon, Bhojghata, Daranchi \\
\hline & Kulbhatti & Kulbhatti, Khedegaon, Fulkoda, Tumdi kasa \\
\hline & Muska & Muska \\
\hline & Devsura & Kosami, Devsura \\
\hline & Pannemara & Pannemara, Ampayali \\
\hline & Yerkad & Muzal gondi, Kanhartola, Tavetola, Sindesur \\
\hline \multirow{3}{*}{ Rangi } & Nimgaon & Borigaon, Nimanwada, Shivgatta \\
\hline & Mohali & Kanargaon \\
\hline & Jangada & Jangada gaon \& Tola \\
\hline \multirow{2}{*}{ Regadi } & Regadi & Regadi, Garnjigaon \& Tola, Vegnoor \\
\hline & Chapalwara & Potepalli \\
\hline \multirow{3}{*}{ Potegaon } & Guruwada & Rakhagaon \& Tola \\
\hline & Maroda & Maroda, Savela \\
\hline & Yewali & Kurpada \\
\hline \multirow{4}{*}{ Amirza } & Kursa & Kursa \\
\hline & Murmadi & Murmadi \\
\hline & Usegaon & Usegaon \\
\hline & Maregaon & Maregaon \& Tola \\
\hline Bodali & Bhagwan pur & Bhagwan pur \\
\hline Mahagaon & Mukhtav pur & Mukhtav pur, Chintal pade, Allapalli, Muktapur \\
\hline
\end{tabular}




\section{Mosquito Collection}

Mosquitoes were collected from various villages of high malaria endemic PHCs of Gadchiroli district of Maharashtra in years 2015-19 to know the susceptibility status of malaria vectors as per the World Health Organization (WHO) procedure. ${ }^{16}$ The selection of study villages of malariaendemic PHCs was based on the prevalence of malaria cases during the years 2011-15 and the availability of vector mosquitoes. Adult mosquitoes were collected from 156 villages of 18 high malaria endemic PHCs out of a total of 47 PHCs of Gadchiroli (Table 2) by using an aspirator and flashlight from human dwellings/ cattle sheds in the morning from 6-9 am including mosquito larvae from different breeding habitats. ${ }^{17}$ An. culicifacies and An. fluviatilis collected were provided $10 \%$ glucose solution soaked in cotton pads and transported in caged cloth to the field laboratory at the PHC and district level. Similarly, mosquito larvae were collected from different breeding sources and reared till emergence for proper identification. ${ }^{18,19}$ During mosquito collection, maximum number of An. culicifacies and An. fluviatilis were recorded in cattle sheds as compared to human dwellings in the surveyed villages.

\section{Bioassay Test}

Bioassay test kits and insecticide-impregnated papers at diagnostic concentrations of various insecticides were received from University Sains Malaysia (vector control unit of WHO). Susceptibility tests were conducted by using the bioassay test kit for lambda cyhalothrin $(0.05 \%)$, permethrin $(0.75 \%)$, cyfluthrin $(0.15 \%)$, deltamethrin $(0.05 \%)$, malathion (5.0\%) and DDT (4.0\%) against An. culicifacies and An. fluviatilis as per WHO procedure. ${ }^{16,17}$ Only full-fed and semi-gravid female mosquitoes were exposed against diagnostic concentrations of different insecticides for one hour as per the WHO procedure. Three to five replicates of 10-25 female mosquitoes were exposed against diagnostic concentrations of each insecticide. Control replicates were also run parallel to each test of insecticide. After exposure, the holding tubes were kept for recovery in dark and cool places immediately under controlled temperature and humidity conditions. Cotton pads soaked in glucose solution were given as supplementary food to the tested mosquitoes during the recovery period for 24 hours.

\section{Interpretation of Results}

The mortality was calculated by counting the number of dead and live mosquitoes after the post-exposure period in both the test and control tubes. The results of this study were made by following criteria, the strain was considered resistant if the mortality was $<80 \%$, verification required/ tolerant/ possible resistant, if mortality ranged between $80-97.99 \%$, and susceptible in case of $98-100 \%$ mortality. If the control mortality was above $5 \%$ but less than $20 \%$, then the observed mortality was corrected by using the following Abbot's formula: ${ }^{20}$

$$
\begin{aligned}
& \text { Observed mortality }=\frac{\text { Total number of dead mosquitoes }}{\text { Total sample size }} \\
& \text { Per cent corrected mortality }=\frac{\% \text { observed mortality }-\% \text { control mortality }}{-\mathrm{x} 100} \\
& 100-\mathrm{o} \text { control mortality }
\end{aligned}
$$

\section{Cone Bioassay Test}

Cone bioassay tests were carried out in 20 sprayed villages of 4 PHCs of Gadchiroli to assess the residual action of insecticide spray on wall surfaces available in the study area by using the WHO bioassay test kit as per WHO procedure. ${ }^{21}$ The houses sprayed with the insecticide on different surfaces were selected for cone bioassay tests. Before starting these tests, the wild-caught full-fed/semigravid female adults of An. culicifacies were collected from unsprayed villages and their $\mathrm{F} 1$ generation was used to determine the efficacy of insecticide. WHO Plastic bioassay cones were attached with self-adhesive packing on sprayed wall surfaces of houses in each village and full-fed/ semigravid 10-20 female mosquitoes were introduced in each plastic bioassay cone for 30 minutes. Each test was done with three replicates with control for a standard period of IRS during morning hours. After 30 minutes of exposure, all the mosquitoes were removed from the bioassay cones and were held in recovery cages at maintained temperature and humidity. Cotton pads soaked in glucose solution were given as supplementary food to the tested mosquitoes during the recovery period for 24 hours and mortality was calculated after 24 hours. This study indicates the efficacy of the insecticide over a period on different wall surfaces.

\section{Results}

Insecticide susceptibility of An. fluviatilis collected from 23 villages showed that all the populations were fully susceptible (98.3-100.0\% mortality) to synthetic pyrethroids (deltamethrin) and resistant (73.8-79.5\% mortality) to organophosphorous malathion (Table 3 ). Susceptibility study of An. culicifacies collected from 156 villages showed that 57 populations were fully susceptible to deltamethrin with a mortality range of $98.0 \%$ to $100.0 \%$ and 96 populations were tolerant to deltamethrin with $80.0 \%$ to $97.5 \%$ mortality. Only three populations of An. culicifacies were resistant to deltamethrin with $77.5 \%$ to $78.0 \%$ mortality (Table 4 and Figure 1). In addition, An. culicifacies collected from 12 same villages was tested against lambda cyhalothrin, out of which 6 populations were found susceptible with from 98.6 to $100.0 \%$ and 6 populations were found tolerant to lambda cyhalothrin with from 85.0 to $97.6 \%$ mortality. Similarly, 18 populations were tested against cyfluthrin, out of which 9 populations were fully susceptible with $100.0 \%$ mortality and 9 populations were tolerant (88.3-96.2\% mortality). 
Further 9 populations were tested against permethrin, out of which 8 populations were fully susceptible with mortality varying from $88.3 \%$ to $100.0 \%$ and only one population was tolerant to permethrin with $97.5 \%$ mortality (Table 4). Resistant was recorded in 25 populations of An. culicifacies to malathion with mortality ranging from $60.0 \%-78.75 \%$ and 30 populations were found to be tolerant to malathion with $80.0 \%$ to $96.2 \%$ mortality (Table 4 and Figure 2 ). All the remaining populations of both species of An. culicifacies and An. fluviatilis were highly resistant to organochlorine (DDT $4.0 \%$ ) with mortality range from $20.0 \%$ to $58.3 \%$ and $43.3 \%$ to $51.2 \%$ respectively as shown in Tables 3 and 4.

Table 3.Insecticide Susceptibility of An. fluviatilis in Gadchiroli district of Maharashtra

\begin{tabular}{|c|c|c|c|c|c|c|c|c|}
\hline \multirow{2}{*}{$\begin{array}{l}\text { Insecticides } \\
\text { Tested \& Dose }\end{array}$} & \multirow{2}{*}{$\begin{array}{c}\text { Name of PHC \& } \\
\text { Name of Villages } \\
\text { surveyed in that PHC }\end{array}$} & \multicolumn{2}{|c|}{$\begin{array}{c}\text { No. of Mosquitoes } \\
\text { Exposed }\end{array}$} & \multicolumn{2}{|c|}{$\begin{array}{c}\text { No. of Mosquitoes } \\
\text { Dead in } 24 \text { Hours }\end{array}$} & \multicolumn{2}{|c|}{ \% Mortality } & \multirow{2}{*}{$\begin{array}{c}\text { Corrected } \\
\text { Mortality (\%) }\end{array}$} \\
\hline & & Exp & Cont & Exp & Cont & Exp & Cont & \\
\hline DDT 4.0 & \multirow{3}{*}{$\begin{array}{c}\text { Kotgul (Sonpur, } \\
\text { Bhimankozi, } \\
\text { Gyarahpatti, Pitesur } \\
\text { gaon \& tola, Nehal } \\
\text { gad) }\end{array}$} & 30 & 10 & 14 & 00 & 46.66 & 00 & 46.66 \\
\hline Malathion 5.0 & & 45 & 15 & 34 & 00 & 75.55 & 6.66 & 73.8 \\
\hline Deltamethrin 0.05 & & 178 & 60 & 178 & 00 & 100 & 3.33 & 100 \\
\hline DDT 4.0 & \multirow{3}{*}{$\begin{array}{l}\text { Malewada (Mendha, } \\
\text { Khobramenda, } \\
\text { Waghabhumi) }\end{array}$} & 30 & 10 & 15 & 00 & 50.00 & 00 & 50.00 \\
\hline Malathion 5.0 & & 44 & 15 & 35 & 00 & 79.54 & 00 & 79.54 \\
\hline Deltamethrin 0.05 & & 180 & 60 & 178 & 00 & 98.88 & 5 & 98.88 \\
\hline DDT 4.0 & \multirow{2}{*}{$\begin{array}{c}\text { Kasansur } \\
\text { (Kondawahi) }\end{array}$} & 30 & 10 & 13 & 00 & 43.33 & 00 & 43.33 \\
\hline Deltamethrin 0.05 & & 60 & 20 & 59 & 00 & 98.33 & 00 & 98.33 \\
\hline Deltamethrin 0.05 & Todsa (Tadhpalli) & 55 & 20 & 55 & 00 & 100 & 5 & 100 \\
\hline Malathion 5.0 & \multirow{2}{*}{ Aarewada (Medpalli) } & 60 & 20 & 47 & 00 & 78.33 & 00 & 78.33 \\
\hline Deltamethrin 0.05 & & 60 & 20 & 60 & 00 & 100 & 00 & 100 \\
\hline Deltamethrin 0.05 & Laheri (Murangal) & 60 & 20 & 60 & 00 & 100 & 5 & 100 \\
\hline DDT 4.0 & \multirow{3}{*}{$\begin{array}{c}\text { Pendhari } \\
\text { (Pekinmudza, Khargi, } \\
\text { Paidi, Gota gaon \& } \\
\text { tola, Jhawal zora) }\end{array}$} & 30 & 10 & 15 & 00 & 50.00 & 00 & 50.00 \\
\hline Malathion 5.0 & & 45 & 15 & 35 & 00 & 77.77 & 6.66 & 76.18 \\
\hline Deltamethrin 0.05 & & 265 & 100 & 264 & 00 & 99.66 & 3 & 99.66 \\
\hline DDT 4.0 & \multirow{3}{*}{$\begin{array}{c}\text { Murumgaon } \\
\text { (Markagaon \& } \\
\text { tola, Makdand, } \\
\text { ongarhud, Devsura, } \\
\text { Fulkodo) } \\
\end{array}$} & 45 & 15 & 26 & 00 & 57.77 & 13.3 & 51.29 \\
\hline Malathion 5.0 & & 45 & 15 & 34 & 00 & 75.55 & 00 & 75.55 \\
\hline Deltamethrin 0.05 & & 290 & 100 & 288 & 00 & 99.33 & 4 & 99.33 \\
\hline DDT 4.0 & \multirow{2}{*}{$\begin{array}{l}\text { Karwafa (Kondawahi } \\
\text { gaon \& tola) }\end{array}$} & 30 & 10 & 13 & 00 & 43.33 & 00 & 43.33 \\
\hline Deltamethrin 0.05 & & 60 & 20 & 60 & 00 & 100 & 00 & 100 \\
\hline
\end{tabular}

The mortality ranges were grouped as: $<80 \%$ (resistant), $80-97.99 \%$ (tolerant), and $98-100 \%$ (susceptible).

Table 4.Insecticide Susceptibility of An. culicifacies in Gadchiroli district of Maharashtra

\begin{tabular}{|c|c|c|c|c|c|c|c|}
\hline \multirow{3}{*}{ Name of PHC } & \multirow{2}{*}{ Name of Village } & \multicolumn{6}{|c|}{ Observed Corrected Mortality (\%) } \\
\cline { 2 - 8 } & & DDT & Mala & Delta & LCM $\mathbf{0 . 0 5}$ & $\begin{array}{c}\text { Cyflu } \\
\mathbf{0 . 1 5}\end{array}$ & $\begin{array}{c}\text { Perm } \\
\mathbf{0 . 7 5}\end{array}$ \\
\hline \multirow{3}{*}{ Kotgul } & Sonpur & - & 73.75 & 98.75 & - & - & - \\
\cline { 2 - 8 } & Bhimankozi & 27.5 & 80.00 & 99.00 & - & - & - \\
\cline { 2 - 8 } & Gyarahpatti & 35.00 & 62.5 & 98.97 & - & - & - \\
\cline { 2 - 8 } & Pitesur gaon \& tola & - & 76.25 & 99.00 & - & 100 & - \\
\cline { 2 - 8 } & Nehalgad & - & - & 100 & - & - & - \\
\cline { 2 - 8 } & Kosami 2 & - & - & 100 & - & - & - \\
\hline
\end{tabular}




\begin{tabular}{|c|c|c|c|c|c|c|c|}
\hline \multirow{12}{*}{ Malewada } & Devsara & - & 81.66 & 90.00 & 85.00 & 100 & - \\
\hline & Mendha & 26.25 & 73.07 & 98.75 & - & 100 & - \\
\hline & Murmadi & - & 95.00 & 95.00 & - & - & - \\
\hline & Khatitola & - & - & 100 & - & - & - \\
\hline & Khobramenda & 31.00 & 70.00 & 99.00 & - & - & - \\
\hline & Huryaldand & - & 82.5 & 98.75 & - & - & - \\
\hline & Badbada & 48.86 & 78.75 & 98.75 & 100 & 100 & - \\
\hline & Yeduskuhi & - & - & 98.75 & 100 & 100 & 100 \\
\hline & Waghabhumi & - & - & 78.00 & - & - & - \\
\hline & Mangewada & - & - & 83.33 & - & - & - \\
\hline & Jaysing tola & - & - & 88.00 & - & 91.11 & - \\
\hline & Khamtala & - & - & 98.00 & 100 & - & - \\
\hline \multirow{9}{*}{ Gatta } & Jhanbia gaon \& tola & - & - & 83.75 & - & - & - \\
\hline & Andenge & 45.00 & 81.11 & 98.88 & - & - & - \\
\hline & Nander & 28.33 & - & 90.00 & - & - & - \\
\hline & Hedari gaon & - & - & 88.00 & - & - & - \\
\hline & Mallam pahari & - & 80.00 & 100 & - & - & - \\
\hline & Surjagad & - & - & 89.00 & - & - & - \\
\hline & Gattagaon \& tola & - & - & 95.00 & - & - & - \\
\hline & Mohndi gaon & - & - & 87.5 & - & - & - \\
\hline & Tadguda & - & - & 97.00 & - & - & - \\
\hline \multirow{9}{*}{ Kasansur } & Kotami & - & - & 90.00 & - & 93.75 & 97.5 \\
\hline & Etawahi & - & - & 93.00 & - & - & - \\
\hline & Kondawahi & - & 91.66 & 100 & - & - & 100 \\
\hline & Tadguda & - & 76.25 & 99.00 & - & - & - \\
\hline & Ghotsur & - & 70.00 & 92.5 & - & - & - \\
\hline & Jaweli & 28.33 & - & 96.00 & - & - & - \\
\hline & Venasur & - & - & 100 & - & - & - \\
\hline & Chokhewada & - & - & 89.00 & - & - & - \\
\hline & Jhuri & 40.00 & 90.00 & 99.00 & 98.6 & 95.00 & 100 \\
\hline \multirow{10}{*}{ Todsa } & Ekrakhurd & - & 93.75 & 100 & - & - & - \\
\hline & Ekrabhurj & - & - & 98.75 & - & - & - \\
\hline & Aalinga gaon \& tola & 33.33 & - & 99.00 & - & - & - \\
\hline & Zarewada & - & - & 100 & - & - & - \\
\hline & Alandi & - & - & 100 & - & - & - \\
\hline & Tadhpalli & - & 65.00 & 91.25 & - & - & - \\
\hline & Lanji & - & - & 92.00 & 100 & - & - \\
\hline & Karampalli gaon \& tola & - & - & 96.92 & - & - & - \\
\hline & Pethagaon & - & - & 92.5 & - & - & - \\
\hline & Devada & 33.33 & - & 100 & - & - & - \\
\hline \multirow{2}{*}{ Aarewada } & Hemal kasha & 26.66 & 75.00 & 100 & - & - & - \\
\hline & Karampalli gaon \& tola & - & - & 100 & - & - & - \\
\hline
\end{tabular}




\begin{tabular}{|c|c|c|c|c|c|c|c|}
\hline & Aarewada & - & - & 96.28 & - & - & - \\
\hline & Medpalli & 38.33 & 93.33 & 100 & - & 100 & 100 \\
\hline & Dubbaguda & - & - & 95.00 & - & - & - \\
\hline & Tadgaon & - & - & 93.75 & - & - & - \\
\hline & Dhulepalli & - & - & 100 & - & - & - \\
\hline & Erakdumme & - & - & 87.5 & - & - & - \\
\hline \multirow{10}{*}{ 7. Laheri } & Dhondraj & - & - & 92.5 & - & - & - \\
\hline & Ranipudar & 35.00 & 91.66 & 92.5 & - & - & - \\
\hline & Kukamenda & - & 95.00 & 100 & - & - & - \\
\hline & Malumpadur & - & - & 100 & & 100 & \\
\hline & Bhoosewada & - & - & 96.66 & - & - & - \\
\hline & Laheri & - & 87.5 & 89.23 & - & - & - \\
\hline & Murangal & - & - & 88.33 & - & - & - \\
\hline & Aaldandi & 31.66 & - & 95.00 & - & - & - \\
\hline & Gundenoor & - & - & 93.75 & - & - & - \\
\hline & Beena gunda & - & 90.00 & 99.00 & - & - & - \\
\hline \multirow{10}{*}{$\begin{array}{l}\text { 8. Manne } \\
\text { Rajaram }\end{array}$} & Mokela & - & - & 100 & - & 100 & - \\
\hline & Chichoda & - & - & 90.00 & - & - & - \\
\hline & Jijgaon & - & - & 93.75 & - & - & - \\
\hline & Bhadenagar & - & - & 100 & - & - & - \\
\hline & Yechali & - & - & 91.66 & - & - & - \\
\hline & Basa guda & 26.66 & 88.33 & 98.33 & - & - & - \\
\hline & Rela & 21.11 & 96.25 & 100 & - & - & - \\
\hline & Rai guda & - & - & 91.66 & - & - & - \\
\hline & Marampalli & - & - & 83.80 & - & - & - \\
\hline & Lakan duga & - & - & 96.25 & - & - & - \\
\hline \multirow{8}{*}{ Godalwahi } & Godalwahi gaon & 31.66 & 80.00 & 93.75 & - & - & - \\
\hline & Godalwahi tola & - & 80.00 & 77.5 & - & - & - \\
\hline & Michgaon & - & - & 96.25 & - & - & - \\
\hline & Sallebhatti & - & 83,75 & 98.75 & 91.25 & - & - \\
\hline & Lekha & 30.00 & 85.00 & 100 & - & - & - \\
\hline & Kannartola & - & - & 98.75 & - & - & - \\
\hline & Mendha & - & - & 95.38 & - & - & - \\
\hline & Udegaon & - & - & 83.33 & - & - & - \\
\hline \multirow{8}{*}{ Pendhari } & Pulkal & - & 81.16 & 96.25 & - & - & - \\
\hline & Dhorgatta & - & - & 96.25 & 85.00 & - & - \\
\hline & Pekinmudza & - & - & 95.00 & - & - & - \\
\hline & Durgapur & 31.66 & - & 97.5 & 90.00 & - & - \\
\hline & Khargi & 33.33 & 83.75 & 100 & 100 & - & - \\
\hline & Chichoda & - & - & 95.00 & - & - & - \\
\hline & Sinsur & - & - & 88.75 & - & - & - \\
\hline & Kaneli & - & - & 98.75 & - & - & - \\
\hline
\end{tabular}


Singh RK et al.

\begin{tabular}{|c|c|c|c|c|c|c|c|}
\hline & Paidi & - & 68.33 & 100 & - & - & - \\
\hline & Gotagaon \& tola & - & 60.00 & 77.5 & - & 91.66 & 100 \\
\hline & Jhawal zora & 24.00 & 71.66 & 98.75 & - & - & - \\
\hline & Pather gatta & - & 71.66 & 86.25 & - & 90.00 & - \\
\hline \multirow{14}{*}{ Karwafa } & Kondawahi gaon \& tola & 26.66 & 76.66 & 97.5 & - & - & - \\
\hline & Fulbodi & - & - & 88.75 & - & - & - \\
\hline & Zari & - & - & 87.5 & - & 88.33 & - \\
\hline & Kanartola & - & - & 91.25 & - & - & - \\
\hline & Sakhera & - & - & 89.13 & - & - & - \\
\hline & Jambli tola & - & - & 94.19 & - & - & - \\
\hline & Pustola & - & - & 94.66 & - & - & - \\
\hline & Kopagoda & - & - & 95.00 & - & - & - \\
\hline & Katezari & - & - & 95.83 & - & - & - \\
\hline & Mendha tola & - & - & 89.33 & - & - & - \\
\hline & Khutgaon & - & - & 90.66 & - & - & - \\
\hline & Michgaon & - & - & 93.75 & - & - & - \\
\hline & Talodhi & - & - & 90.00 & - & - & - \\
\hline & Navegaon & 36.66 & 84.12 & 98.75 & 97.46 & - & - \\
\hline \multirow{24}{*}{ Murumgaon } & Morchul & - & - & 93.47 & 91.66 & - & - \\
\hline & Savar gaon & - & - & 96.25 & - & - & - \\
\hline & Markagaon \& tola & 58.33 & 73.75 & 98.75 & - & - & - \\
\hline & Gajamendi & - & - & 97.5 & - & 100 & - \\
\hline & Kangadi & - & - & 93.75 & - & - & - \\
\hline & Makdand & - & - & 96.25 & - & - & - \\
\hline & Tadegaon & - & - & 98.75 & - & - & - \\
\hline & Dongarhud & - & - & 100 & - & - & - \\
\hline & Sursundi & 38.33 & 82.5 & 93.75 & - & - & - \\
\hline & Eurup dodari & - & - & 92.5 & - & - & - \\
\hline & Saygaon & - & - & 100 & - & - & - \\
\hline & Bhojghata & - & - & 100 & - & - & - \\
\hline & Daranchi & - & - & 100 & - & - & - \\
\hline & Kosami & - & - & 93.00 & - & - & - \\
\hline & Devsura & - & 85.00 & 96.25 & - & - & - \\
\hline & Kulbhatti & 50.00 & 75.00 & 97.09 & - & - & - \\
\hline & Khedegaon & - & 78.75 & 92.5 & - & - & - \\
\hline & Fulkoda & 23.63 & 78.33 & 95.23 & - & 92.85 & 98.33 \\
\hline & Tumdi kasa & - & 75.00 & 94.56 & - & - & - \\
\hline & Pannemara & 33.33 & - & 85.00 & - & - & - \\
\hline & Ampayali & - & - & 91.25 & - & - & - \\
\hline & Muzalgondi & 26.66 & - & 90.00 & & 93.33 & 100 \\
\hline & Kanhartola & - & - & 81.25 & - & - & - \\
\hline & Tavetola & & 72.5 & 97.5 & - & - & - \\
\hline
\end{tabular}




\begin{tabular}{|c|c|c|c|c|c|c|c|}
\hline & Sindesur & - & - & 96.25 & - & - & - \\
\hline & Muska & - & - & 94.44 & - & - & - \\
\hline \multirow{5}{*}{ Rangi } & Borigaon & 28.33 & 71.66 & 83.75 & - & - & - \\
\hline & Niman wada & - & - & 85.00 & - & - & - \\
\hline & Shivgatta & - & - & 88.33 & - & - & - \\
\hline & Kanar gaon & - & - & 88.75 & - & - & - \\
\hline & Jangada gaon \& tola & 50.00 & 75.00 & 90.00 & - & - & - \\
\hline \multirow{4}{*}{ Regadi } & Regadi & 21.66 & - & 91.25 & - & - & - \\
\hline & Garnji gaon \& tola & - & 72.5 & 87.5 & - & - & - \\
\hline & Vegnoor & - & - & 95.38 & - & - & - \\
\hline & Potepalli & 55.00 & 80.00 & 90.00 & - & - & - \\
\hline \multirow{4}{*}{ Potegaon } & Rakha gaon \& tola & 30.00 & 80.00 & 100 & - & 96.25 & 100 \\
\hline & Maroda & - & - & 93.75 & - & - & - \\
\hline & Savela & - & - & 96.66 & - & - & - \\
\hline & Kurpada & - & - & 98.75 & - & - & - \\
\hline \multirow{4}{*}{ Amirza } & Kursa & 20.00 & 86.75 & 96.25 & - & - & - \\
\hline & Murmadi & - & - & 95.00 & - & - & - \\
\hline & Usegaon & 32.00 & 87.5 & 100 & - & - & - \\
\hline & Maregaon \& tola & - & - & 97.33 & - & - & - \\
\hline Bodali & Bhagwan pur & 25.00 & 66.66 & 99.00 & - & - & - \\
\hline \multirow{4}{*}{ Mahagaon } & Mukhtav pur & 25.00 & 86.66 & 96.66 & - & - & - \\
\hline & Chintal pade & - & - & 98.66 & - & - & - \\
\hline & Allapalli & - & - & 100 & - & - & - \\
\hline & Muktapur & - & - & 98.75 & - & - & - \\
\hline
\end{tabular}

The mortality ranges were grouped as: $<80 \%$ (resistant), $80-97.99 \%$ (tolerant), and $98-100 \%$ (susceptible).

The PHC-wise susceptibility of An. culicifacies was analysed and observed. The corrected mortality range in different PHCs varied from $18.00 \%$ to $58.38 \%$ for DDT, $60.00 \%$ to $96.25 \%$ for malathion, $77.5 \%$ to $100.0 \%$ for deltamethrin, $85.00 \%$ to $100.00 \%$ for lambda cyhalothrin, $88.33 \%$ to $100.00 \%$ for cyfluthrin and $97.5 \%$ to $100.00 \%$ for permethrin (Table 5). The population of An. culicifacies was fully susceptible to deltamethrin in Kotgul and Bodali PHC, tolerant in 16 other PHCs surveyed, and resistant to deltamethrin in only three villages of three PHCs of Gadchiroli (Figure 1). Results of the present study showed that the main malaria vector An. culicifacies was highly resistant to DDT in all the PHCs of Gadchiroli (Figure 3). Resistance to malathion in An. culicifacies was recorded in 11 PHCs and tolerance to malathion was recorded in 7 PHCs in the study area. In addition, An. culicifacies species was not found resistant to other synthetic pyrethroids (lambda cyhalothrin, cyfluthrin and permethrin) in any PHC of Gadchiroli, but the species was found tolerant to lambda cyhalothrin and cyfluthrin in $6 \mathrm{PHCs}$, and tolerant to permethrin in only one village of one PHC, Kasansur.

Table 5.Primary Health Centre-wise Insecticide Susceptibility of An. culicifacies in Gadchiroli district of Maharashtra

\begin{tabular}{|c|c|c|c|c|c|c|c|c|c|}
\hline \multirow{2}{*}{$\begin{array}{c}\text { Insecticides tested \& } \\
\text { dose (\%) }\end{array}$} & \multirow{2}{*}{$\begin{array}{l}\text { Name of } \\
\text { PHC (No. } \\
\text { of Villages } \\
\text { surveyed in } \\
\text { that PHC) }\end{array}$} & \multicolumn{2}{|c|}{$\begin{array}{c}\text { No. of } \\
\text { Mosquitoes } \\
\text { Exposed }\end{array}$} & \multicolumn{2}{|c|}{$\begin{array}{c}\text { No. of } \\
\text { Mosquitoes Dead } \\
\text { in } 24 \text { Hours }\end{array}$} & \multicolumn{2}{|c|}{$\begin{array}{l}\text { Mortality } \\
\text { (\%) }\end{array}$} & \multirow{2}{*}{$\begin{array}{c}\text { Corrected } \\
\text { Mortality } \\
\text { (\%) }\end{array}$} & \multirow{2}{*}{$\begin{array}{c}\text { \% Corrected } \\
\text { Mortality } \\
\text { Range in } \\
\text { PHC } \\
\end{array}$} \\
\hline & & Exp & ont & Exp & ont & $\mathrm{xp}$ & tt & & \\
\hline$\Delta$ & \multirow[b]{2}{*}{ (bul } & 0 & & & & U2.. & & 62 & $27.5-35.00$ \\
\hline alathion 5 & & 160 & 40 & 111 & 00 & 69.37 & 00 & 69.37 & $62.5-80.00$ \\
\hline
\end{tabular}


Singh RK et al.

\begin{tabular}{|c|c|c|c|c|c|c|c|c|c|}
\hline Deltamethrin 0.05 & & 560 & 135 & 556 & 05 & 99.25 & 3.70 & 99.25 & $98.75-100$ \\
\hline Cyfluthrin 0.15 & & 80 & 20 & 80 & 00 & 100 & 00 & 100 & 100 \\
\hline DDT 4.0 & \multirow{6}{*}{$\begin{array}{l}\text { Malewada } \\
\quad(12)\end{array}$} & 270 & 70 & 97 & 03 & 36.92 & 4.28 & 36.92 & $26.25-48.86$ \\
\hline Malathion 5.0 & & 400 & 110 & 307 & 06 & 76.75 & 5.45 & 75.35 & $73.07-82.5$ \\
\hline Deltamethrin 0.05 & & 1030 & 225 & 960 & 06 & 93.20 & 2.66 & 93.20 & $78.00-100$ \\
\hline $\begin{array}{l}\text { Lambda cyhalothrin } \\
0.05\end{array}$ & & 380 & 95 & 367 & 01 & 96.57 & 1.05 & 96.57 & $85.00-100$ \\
\hline Cyfluthrin 0.15 & & 415 & 105 & 397 & 03 & 95.66 & 2.85 & 95.66 & $91.11-100$ \\
\hline Permethrin 0.75 & & 90 & 20 & 90 & 00 & 100 & 00 & 100 & 100 \\
\hline DDT 4.0 & \multirow{3}{*}{ Gatta (9) } & 140 & 40 & 53 & 00 & 37.85 & 00 & 37.85 & $28.33-45.00$ \\
\hline Malathion 5.0 & & 180 & 40 & 137 & 01 & 97.85 & 2.5 & 97.85 & $80.00-81.11$ \\
\hline Deltamethrin 0.05 & & 830 & 205 & 767 & 04 & 92.40 & 1.95 & 92.40 & $83.75-100$ \\
\hline DDT 4.0 & \multirow{6}{*}{ Kasansur (9) } & 120 & 40 & 39 & 00 & 32.5 & 00 & 32.5 & $28.33-40.00$ \\
\hline Malathion 5.0 & & 280 & 80 & 226 & 00 & 80.71 & 00 & 80.71 & $70.00-90.00$ \\
\hline Deltamethrin 0.05 & & 840 & 210 & 802 & 06 & 95.47 & 2.85 & 95.47 & $89.00-100$ \\
\hline $\begin{array}{c}\text { Lambda cyhalothrin } \\
0.05 \\
\end{array}$ & & 75 & 25 & 74 & 01 & 98.66 & 4.00 & 98.66 & 98.6 \\
\hline Cyfluthrin 0.15 & & 160 & 45 & 152 & 01 & 95.00 & 2.22 & 95.00 & $95.00-95.00$ \\
\hline Permethrin 0.75 & & 220 & 60 & 218 & 00 & 99.09 & 00 & 99.09 & $97.5-100$ \\
\hline DDT 4.0 & \multirow{3}{*}{ Todsa (10) } & 120 & 40 & 35 & 00 & 29.16 & 00 & 29.16 & $33.33-33.33$ \\
\hline Malathion 5.0 & & 240 & 65 & 206 & 01 & 85.83 & 1.53 & 85.83 & $65.00-93.75$ \\
\hline Deltamethrin 0.05 & & 805 & 230 & 788 & 06 & 97.88 & 2.6 & 97.88 & $91.25-100$ \\
\hline DDT 4.0 & \multirow{5}{*}{ Aarewada (8) } & 120 & 40 & 39 & 00 & 32.5 & 00 & 32.5 & $26.66-38.33$ \\
\hline Malathion 5.0 & & 140 & 40 & 126 & 00 & 90.00 & 00 & 90.00 & $75.00-93.33$ \\
\hline Deltamethrin 0.05 & & 620 & 170 & 599 & 04 & 96.61 & 2.35 & 96.61 & $87.5-100$ \\
\hline Cyfluthrin 0.15 & & 80 & 20 & 80 & 00 & 100 & 00 & 100 & 100 \\
\hline Permethrin 0.75 & & 80 & 20 & 80 & 00 & 100 & 00 & 100 & 100 \\
\hline DDT 4.0 & \multirow{4}{*}{ Laheri (10) } & 120 & 40 & 40 & 00 & 33.33 & 00 & 33.33 & $31.66-35.00$ \\
\hline Malathion 5.0 & & 280 & 80 & 254 & 02 & 90.71 & 2.5 & 90.71 & $87.5-95.00$ \\
\hline Deltamethrin 0.05 & & 745 & 205 & 711 & 04 & 95.43 & 1.95 & 95.43 & $88.33-100$ \\
\hline Cyfluthrin 0.15 & & 80 & 20 & 80 & 00 & 100 & 00 & 100 & 100 \\
\hline DDT 4.0 & \multirow{4}{*}{$\begin{array}{c}\text { Manne } \\
\text { Rajaram (10) }\end{array}$} & 120 & 40 & 27 & 02 & 22.5 & 5.00 & 22.5 & $21.11-26.66$ \\
\hline Malathion 5.0 & & 140 & 40 & 130 & 00 & 92.85 & 00 & 92.85 & $88.33-96.25$ \\
\hline Deltamethrin 0.05 & & 695 & 200 & 671 & 04 & 96.54 & 2.00 & 96.54 & $83.80-100$ \\
\hline Cyfluthrin 0.15 & & 60 & 20 & 60 & 00 & 100 & 00 & 100 & 100 \\
\hline DDT 4.0 & \multirow{4}{*}{$\begin{array}{l}\text { Godalwahi } \\
\text { (8) }\end{array}$} & 120 & 40 & 35 & 00 & 29.16 & 00 & 29.16 & $30.00-31.66$ \\
\hline Malathion 5.0 & & 320 & 80 & 263 & 01 & 82.18 & 1.25 & 82.18 & $80.00-85.00$ \\
\hline Deltamethrin 0.05 & & 600 & 150 & 552 & 02 & 92.00 & 1.33 & 92.00 & $77.5-100$ \\
\hline $\begin{array}{l}\text { Lambda cyhalothrin } \\
0.05\end{array}$ & & 80 & 20 & 74 & 00 & 92.5 & 00 & 92.5 & 91.25 \\
\hline DDT 4.0 & \multirow{3}{*}{ Pendhari (12) } & 195 & 65 & 57 & 01 & 29.23 & 1.53 & 29.23 & $18.00-33.33$ \\
\hline Malathion 5.0 & & 400 & 120 & 291 & 03 & 72.75 & 2.5 & 72.75 & $60.00-83.75$ \\
\hline Deltamethrin 0.05 & & 860 & 220 & 798 & 06 & 92.79 & 2.72 & 92.79 & $77.5-100$ \\
\hline
\end{tabular}




\begin{tabular}{|c|c|c|c|c|c|c|c|c|c|}
\hline $\begin{array}{c}\text { Lambda cyhalothrin } \\
0.05\end{array}$ & & 198 & 60 & 183 & 00 & 92.42 & 00 & 92.42 & $85.00-100$ \\
\hline Cyfluthrin 0.15 & & 60 & 20 & 55 & 00 & 91.66 & 00 & 91.66 & $90.00-100$ \\
\hline Permethrin 0.75 & & 70 & 20 & 70 & 00 & 100 & 00 & 100 & 100 \\
\hline DDT 4.0 & \multirow{5}{*}{ Karwafa (14) } & 120 & 40 & 38 & 00 & 31.66 & 00 & 31.66 & $26.66-36.33$ \\
\hline Malathion 5.0 & & 203 & 60 & 165 & 01 & 81.28 & 1.66 & 81.28 & $76.66-84.75$ \\
\hline $\begin{array}{l}\text { Deltamethrin } \\
0.05\end{array}$ & & 1100 & 295 & 1019 & 06 & 9263 & 2.03 & 9263 & $87.5-98.75$ \\
\hline $\begin{array}{c}\text { Lambda cyhalothrin } \\
0.05 \\
\end{array}$ & & 79 & 20 & 77 & 00 & 97.46 & 00 & 97.46 & 97.46 \\
\hline Cyfluthrin 0.15 & & 60 & 20 & 53 & 00 & 88.33 & 00 & 88.33 & 88.33 \\
\hline DDT 4.0 & \multirow{6}{*}{$\begin{array}{l}\text { Murumgaon } \\
(26)\end{array}$} & 360 & 125 & 140 & 00 & 38.88 & 00 & 38.88 & $23.63-58.38$ \\
\hline Malathion 5.0 & & 620 & 165 & 483 & 02 & 77.99 & 1.21 & 77.99 & $73.75-85.00$ \\
\hline Deltamethrin 0.05 & & 2080 & 545 & 2043 & 14 & 98.22 & 2.56 & 98.22 & $81.25-100$ \\
\hline $\begin{array}{c}\text { Lambda cyhalothrin } \\
0.05 \\
\end{array}$ & & 60 & 20 & 55 & 00 & 91.66 & 00 & 91.66 & 91.66 \\
\hline Cyfluthrin 0.15 & & 212 & 60 & 205 & 00 & 96.69 & 00 & 96.69 & $92.85-100$ \\
\hline Permethrin 0.75 & & 120 & 40 & 119 & 00 & 99.16 & 00 & 99.16 & $98.33-100$ \\
\hline DDT 4.0 & \multirow{3}{*}{ Rangi (5) } & 120 & 40 & 47 & 00 & 39.16 & 00 & 39.16 & $28.33-50.00$ \\
\hline Malathion 5.0 & & 120 & 40 & 89 & 00 & 74.16 & 00 & 74.16 & $71.66-90.00$ \\
\hline $\begin{array}{l}\text { Deltamethrin } \\
0.05\end{array}$ & & 380 & 100 & 333 & 02 & 87.63 & 2.00 & 87.63 & $83.75-90.00$ \\
\hline DDT 4.0 & \multirow{3}{*}{ Regadi (4) } & 120 & 40 & 46 & 00 & 38.33 & 00 & 38.33 & $21.66-55.00$ \\
\hline Malathion 5.0 & & 140 & 40 & 110 & 00 & 78.57 & 00 & 78.57 & $77.5-80.00$ \\
\hline Deltamethrin 0.05 & & 310 & 80 & 282 & 02 & 90.96 & 2.5 & 90.96 & $87.5-95.38$ \\
\hline DDT 4.0 & \multirow{5}{*}{ Potegaon (4) } & 60 & 20 & 18 & 00 & 30.00 & 00 & 30.00 & 30.00 \\
\hline Malathion 5.0 & & 60 & 20 & 48 & 00 & 80.00 & 00 & 80.00 & 80.00 \\
\hline $\begin{array}{c}\text { Deltamethrin } \\
0.05 \\
\end{array}$ & & 330 & 85 & 321 & 02 & 97.27 & 2.35 & 97.27 & $93.75-100$ \\
\hline $\begin{array}{c}\text { Cyfluthrin } \\
0.15\end{array}$ & & 80 & 20 & 78 & 00 & 97.5 & 00 & 97.5 & 96.25 \\
\hline Permethrin 0.75 & & 80 & 20 & 80 & 00 & 100 & 00 & 100 & 100 \\
\hline DDT 4.0 & \multirow{3}{*}{ Amirza (4) } & 110 & 40 & 30 & 00 & 27.27 & 00 & 27.27 & 20.32 \\
\hline Malathion 5.0 & & 160 & 40 & 139 & 00 & 86.87 & 00 & 86.87 & $86.75-100$ \\
\hline $\begin{array}{c}\text { Deltamethrin } \\
0.05 \\
\end{array}$ & & 315 & 85 & 306 & 01 & 97.14 & 1.17 & 97.14 & $95.00-100$ \\
\hline DDT 4.0 & \multirow{3}{*}{ Bodali (1) } & 60 & 20 & 15 & 00 & 25.00 & 00 & 25.00 & 25.00 \\
\hline Malathion 5.0 & & 60 & 20 & 40 & 00 & 66.66 & 00 & 66.66 & 66.66 \\
\hline Deltamethrin 0.05 & & 100 & 20 & 99 & 00 & 99.00 & 00 & 99.00 & 98.75 \\
\hline DDT 4.0 & \multirow{3}{*}{$\begin{array}{l}\text { Mahagaon } \\
\text { (4) }\end{array}$} & 60 & 20 & 15 & 00 & 25.00 & 00 & 25.00 & 25.00 \\
\hline Malathion 5.0 & & 60 & 20 & 52 & 00 & 86.66 & 00 & 86.66 & 86.66 \\
\hline Deltamethrin 0.05 & & 275 & 85 & 271 & 01 & 98.54 & 1.17 & 98.54 & $86.66-100$ \\
\hline
\end{tabular}

The mortality ranges were grouped as: $<80$ (resistant), $80-97.99 \%$ (tolerant), and $98-100 \%$ (susceptible). 
Table 6.Cone Bioassay Tests of An. culicifacies on different types of Wall Surfaces sprayed with Lambda cyhalothrin in Study Villages of Malaria Endemic PHCs of Gadchiroli district, Maharashtra

\begin{tabular}{|c|c|c|c|c|c|}
\hline \multirow[t]{2}{*}{$\begin{array}{l}\text { Sr. } \\
\text { No. }\end{array}$} & \multirow[t]{2}{*}{ Name of Village and PHC } & \multirow[t]{2}{*}{ Week } & \multirow[t]{2}{*}{$\begin{array}{l}\text { \% Mortality of } \\
\text { An. culicifacies } \\
\text { in Control }\end{array}$} & \multicolumn{2}{|c|}{$\begin{array}{l}\text { \% Mortality of An. culicifacies on Wall Surfaces of } \\
\text { Sprayed Houses in Study VillageS against Lambda } \\
\text { cyhalothrin } 0.05 \%\end{array}$} \\
\hline & & & & Cemented wall & Mud wall \\
\hline 1. & Kotami (Kasansur) & 2 & $0.00(10)$ & $46.66(30)$ & - \\
\hline 2. & Ghotsur (Kasansur) & 1 & $0.00(10)$ & - & $40.00(30)$ \\
\hline 3. & Kondawahi (Kasansur) & 4 & $0.00(15)$ & - & $28.88(45)$ \\
\hline 4. & Venasur (Kasansur) & 3 & $0.00(10)$ & - & $36.66(30)$ \\
\hline 5. & Chokhewada (Kasansur) & 2 & $0.00(10)$ & - & $33.33(30)$ \\
\hline 6. & Katezari (Murumgaon) & 1 & $6.66(15)$ & $51.11(45)$ & - \\
\hline 7. & Murumgaon (Murumgaon) & 1 & $6.66(15)$ & $48.88(45)$ & - \\
\hline 8. & Savargaon (Murumgaon) & 2 & $0.00(15)$ & $46.66(30)$ & $43.33(30)$ \\
\hline 9. & Markagaon (Murumgaon) & 1 & $0.00(15)$ & - & $27.5(40)$ \\
\hline 10. & Gajamendi (Murumgaon) & 1 & $0.00(10)$ & - & $40.00(30)$ \\
\hline 11. & Morchul (Murumgaon) & 2 & $0.00(15)$ & - & $31.11(45)$ \\
\hline 12. & Dhorgatta (Pendhari) & 1 & $6.66(15)$ & - & $42.5(40)$ \\
\hline 13. & Pekinmudza (Pendhari) & 1 & $0.00(10)$ & - & $43.33(30)$ \\
\hline 14. & Durgapur (Pendhari) & 2 & $0.00(10)$ & $43.33(30)$ & - \\
\hline 15. & Khargi (Pendhari) & 3 & $0.00(15)$ & $43.33(30)$ & $30.00(30)$ \\
\hline 16. & Paidi (Pendhari) & 2 & $5.00(20)$ & $48.88(45)$ & $33.33(30)$ \\
\hline 17. & Devsara (Malewada) & 1 & $6.66(15)$ & $46.66(30)$ & $27.5(40)$ \\
\hline 18. & Khobra menda (Malewada) & 2 & $5.00(20)$ & $43.33(30)$ & $30.00(30)$ \\
\hline 19. & Jaysing tola (Malewada) & 3 & $0.00(10)$ & $32.5(40)$ & - \\
\hline 20. & Mange wada (Malewada) & 2 & $0.00(10)$ & - & $30.00(30)$ \\
\hline & Mortality range & & $5.00-6.66$ & $51.11-32.5$ & $43.33-27.5$ \\
\hline
\end{tabular}

Note: Figures in brackets represent names of PHCs of Gadchiroli district, Maharashtra and the total number of An. culicifacies tested.

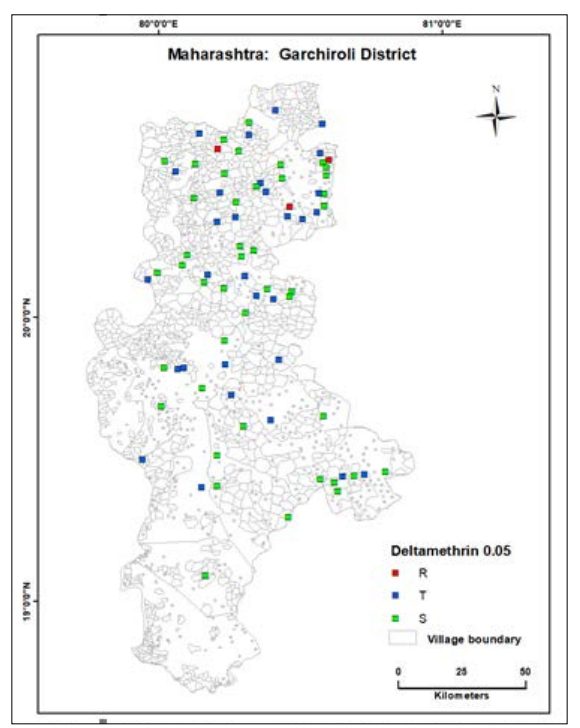

Figure I.Map of Garchiroli District showing Susceptibility Status of Malaria Vector to Deltamethrin in Study Villages of Garchiroli, Maharashtra 


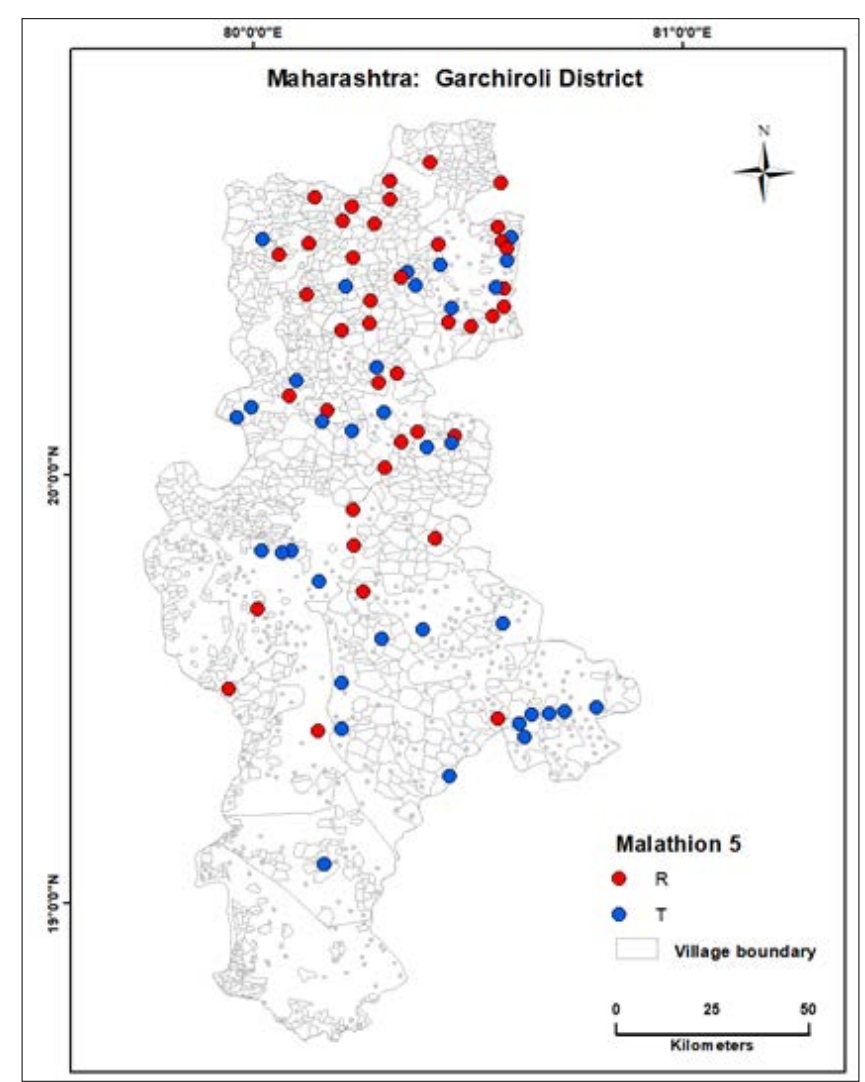

Figure 2.Map of Garchiroli District showing Malathion Resistance \& Tolerance of Malaria Vector in Study Villages of Garchiroli, Maharashtra

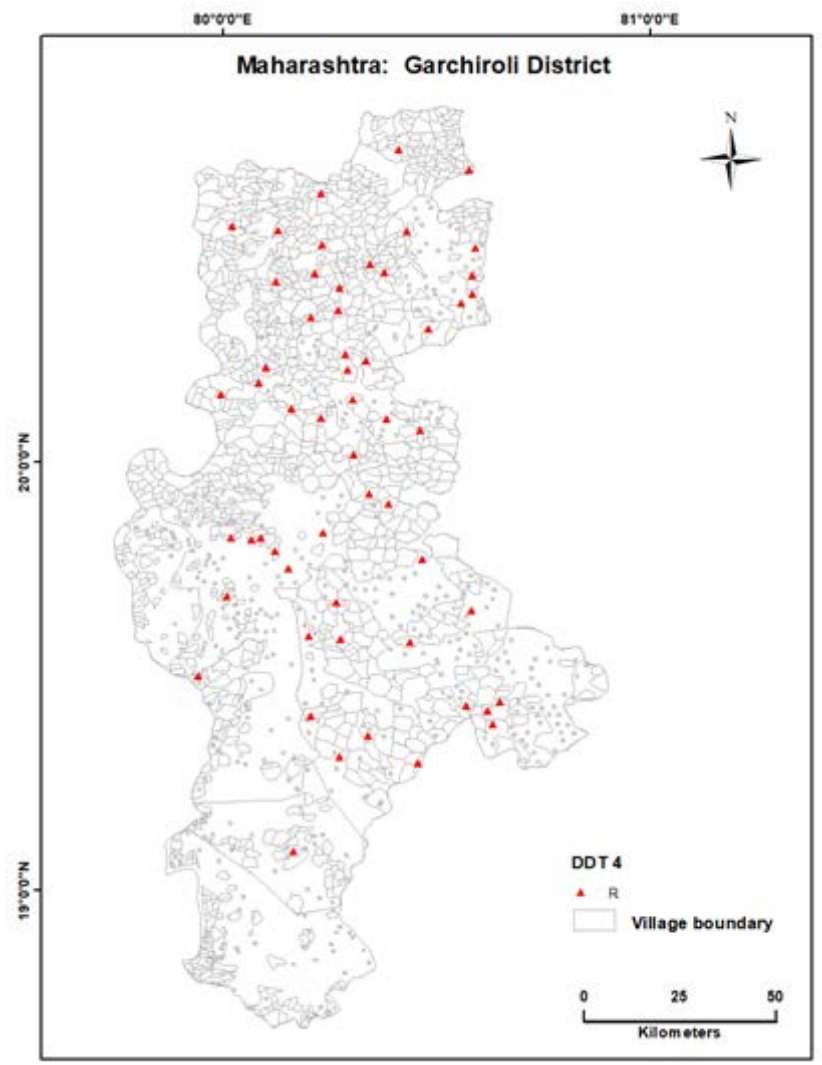

Figure 3.Map of Garchiroli District showing DDT Resistance to Malaria Vector in Study Villages of Garchiroli, Maharashtra 
Residual action of sprayed insecticide was determined by using cone bioassays kits on different sprayed surfaces available in the study area. Results of the cone bioassay tests revealed that IRS was found satisfactory in few sites in terms of effectiveness in the control of An. culicifacies on the wall surfaces tested in 20 study villages of 4 PHCs of Gadchiroli. Mortality range was from $51.11 \%$ to $32.5 \%$ on cemented or $43.33 \%$ to $27.5 \%$ on the mud wall sprayed with lambda cyhalothrin. Lambda cyhalothrin was found more effective on cemented walls as compared to mud walls of the houses. Detailed information regarding the results of cone bioassays is given in Table 6. Quality and coverage of IRS were not found appropriate during the study because IRS coverage was patchy, not uniform, and incomplete on all walls of the houses. Moreover, the spray was found only on upper portion of the outer wall of the houses in some villages.

\section{Discussion}

Malaria control in the country is undertaken by following the guidelines provided by NVBDCP and management of malaria vector is the main tool of the malaria control programme. To manage the malaria vectors, insecticide based intervention measures like IRS and ITBNs/ LLINs are the important components of the Indian malaria control programme, ${ }^{6}$ but the development of insecticide resistance in malaria vectors is one of the major problems in malaria control. IRS with synthetic insecticides like DDT and BHC was used to control malaria vectors for interrupted transmission of malaria in Gadchiroli since 1958. This mode of vector control observed huge success in the beginning, but later development of insecticide resistance in malaria vectors was recorded in 1959 after more than one and a half decades of its use due to continuous use of insecticides under the programme.

An. culicifacies is the common primary vector of malaria responsible for most of malaria epidemics and perennial malaria transmission in India. In Gadchiroli district of Maharashtra, the prevalent vectors of malaria are $A n$. culicifacies and An. fluviatilis, of which An. culicifacies is prevalent widely while An. fluviatilis is prevalent only in few pockets. The findings are in accordance with the studies undertaken from Godda and Gumla districts of Jharkhand, ${ }^{22,23}$ and are also from Murumgaon PHC area of Gadchiroli district. ${ }^{24}$ Dhiman et al. reported An. culicifacies, An. fluviatilis, and An. annularis vectors of malaria from Dhanora taluka of Gadchiroli district and also observed that there was no evidence of outdoor resting of An. culicifacies except in one village of Dhanora PHC in 2005, where the malaria vector was found during hand collections from tree holes near human dwellings. However, An. culicifacies could not be collected in light trap collections. ${ }^{25}$ The finding has given an indication of more possibilities of outdoor malaria transmission as tribal people go to the forest for their earning in early morning hours and come back late. It may play a great role in malaria control.

The resistance in An. culicifacies malaria vector against DDT was first time found in Maharashtra, 2627 and confirmatory report on DDT resistance in An. culicifacies was published by Rahman et al. in the year $1959 .{ }^{10}$ Similarly, DDT resistance in An. culicifacies and An. annularis was reported from a village of Meerut city, Uttar Pradesh in $1962 .{ }^{28}$ Multiple resistance in An. culicifacies to DDT, $\mathrm{HCH}$, and malathion was reported from Thane district of Maharashtra, ${ }^{29}$ and An. culicifacies resistance to $\mathrm{BHC}$ and dieldrin was reported from adjoining areas of Gujarat and Maharashtra, ${ }^{30}$ and other states by earlier research workers. ${ }^{31-32}$ Malathion was introduced in 1969 in IRS for vector control, and resistance in An. culicifacies against malathion was found very quickly from Gujarat within four years of usage of insecticide11; and also from Maharashtra ${ }^{28}$ and other states like Andhra Pradesh, Orissa, Madhya Pradesh, Chhattisgarh and Jharkh and $^{22,31-36}$ Later on, deltamethrin was introduced in IRS in 1996 to control malaria vectors due to their safety for humans at low dosage, excito-repellent properties, and knock-down, killing effects. Deltamethrin was introduced in IRS to control malaria vectors on the pilot basis in Gadchiroli district in 1998, and later in 2008, the insecticide was widely used in IRS activities under malaria control programme, impregnation of bed nets, and making LLINs to prevent malaria. ${ }^{12,13}$

In earlier studies, all the species of An. culicifacies, An. fluviatilis, and $A n$. annularis mosquitoes were found tolerant to deltamethrin and malathion and highly resistant against DDT in Murumgaon PHC of Gadchiroli in the year $2012^{24}$ and later on, Gyan Chand et al. (2017) reported resistance to DDT, malathion and other synthetic pyrethroids as lambda cyhalothrin, deltamethrin, cyfluthrin, and tolerance to permethrin in An. culicifacies. ${ }^{37}$ However, An. culicifacies species has developed resistance to DDT in 286 districts, to Malathion in 81, and to pyrethroids in 2 districts in India. ${ }^{6-8}$ Anopheles fluviatilis is a secondary malaria vector in Gadchiroli district with less prevalence limited to a few villages. However, it has been reported as a primary vector of malaria in the adjoining areas of the neighbouring states of Chhattisgarh and Maharashtra. ${ }^{9,30,38}$

Anopheles fluviatilis plays a role in malaria transmission throughout the year and is mainly found in forest hilly tract villages near a stream. Presently An. fluviatilis is resistant to DDT in 11 districts from 8 states including Maharashtra due to exposure to the pesticides used in agriculture. Resistance in An. fluviatilis against DDT was found from Odisha ${ }^{39}$ and other studies also reported resistance in An. fluviatilis to DDT in areas like Jharkhand and Maharashtra. ${ }^{22,24,31}$ These findings have also been confirmed recently from Gadchiroli 
by Gyan Chand et al. ${ }^{37}$ Similar results have been reported earlier by different researchers from abroad on multiple resistances in An. gambiae, An. funestus, An. arabiensis, and $A n$. coluzzi from different parts of Africa and other countries. But, Sharma et al. (2004) reported that this species was found susceptible to DDT and other insecticides like malathion and deltamethrin in 7 districts of Odisha. ${ }^{32}$ The resistance developed in An. fluviatilis against DDT may be due to the regular use of DDT in IRS since 1958. Later on, the tolerance or resistance of An. fluviatilis to Malathion was found from different districts and states including Maharashtra. $22,24,25,31,32$

The results of the present study indicated that both $A n$. culicifacies and An. fluviatilis were highly resistant to DDT in all the PHCs while moderately resistant to malathion and tolerant to deltamethrin. Similar observations were made by earlier researchers from Madhya Pradesh, central India in the year 2012. ${ }^{35}$ An. culicifacies was found resistant to deltamethrin in a few villages only but susceptible to synthetic pyrethroids in most of the villages. Moreover, it was resistant to malathion in most of the villages of PHCs surveyed and highly resistant to DDT in all the villages. Anopheles fluviatilis was resistant to DDT only, tolerant to malathion and fully susceptible to deltamethrin in all the villages of Gadchiroli district. Cone bioassay tests were done for the determination of residual action in 20 villages of 4 malaria endemic PHCs on the wall surface of the houses sprayed with lambda cyhalothrin insecticide after 1 to 4 weeks of indoor residual spray (IRS). Results of this study revealed that IRS was found satisfactory in terms of effectiveness in the control of An. culicifacies on the wall surfaces tested in 20 study villages of 4 PHCs of Gadchiroli. Lambda cyhalothrin was found more effective on cemented walls as compared to mud walls of the houses and IRS was found satisfactory in a few villages. IRS was not uniform and was found incomplete or patchy on walls of the houses and the quality of IRS was poor in some houses. Similar findings were observed by previous research workers from Odisha and Bhojpur PHC of Moradabad District of Uttar Pradesh by Gunasekaran and Shukla et al. and also from Aurangabad town of Maharashtra state by Vittal et al..$^{45-47}$

\section{Suitable Strategy for Control of Malaria Vectors in Gadchiroli District}

The district of Gadchiroli has been receiving two rounds of IRS with lambda cyhalothrin and cyfluthrin synthetic pyrethroid along with the use of LLINs containing permethrin (Olyset brand) to effectively control malaria. In spite of these control measures, malaria remains a major public health problem in this district. There is an urgent need of an increased emphasis on regular and effective IRS in high-risk PHCs for better control of malaria vectors. PHC wise IRS activities should be done with more susceptible insecticides. The results of the present study may be made use of regarding the selection of the insecticide as malaria vector was resistant to deltamethrin only in few villages. Introduction of newly developed LLINs (with added synergists like PBO) may lead to effective control of the resistant population of mosquito vectors. Moreover, the use of available mosquito repellents should be encouraged in the community to minimise the outdoor biting of malaria vectors since there may be more possibilities of outdoor malaria transmission as most of the tribal people/ villagers go to forests early in the morning to earn their livelihood and come back late. It may play a great role in the control of malaria in the district. There is also a need to find out alternate insecticides for IRS activities and insecticide susceptibility needs to be tested regarding other insecticides of synthetic pyrethroids like bifenthrin \& alpha-cypermethrin as approved by NVBDCP. IRS for these insecticides has not been used before in Gadchiroli district of Maharashtra and it may be a useful option after indication of cross-resistance. Special IEC activities should be taken up in the local language to increase the community's knowledge and awareness and to ensure a change of behaviour at the ground level to eliminate malaria from Gadchiroli district of Maharashtra.

\section{Conclusion}

In conclusion, the finding of this study indicates that resistance has developed to synthetic pyrethroids in the major malaria vector An. culicifacies. As synthetic pyrethroids are used in malaria control programmes for IRS and impregnation of bed nets, it is an adverse indication to achieve the goal of eliminating malaria by 2030 . Therefore, there is an urgent need for the evaluation of new insecticide molecules for better control of malaria vectors. In addition, there is also a need for study on insecticide susceptibility of malaria vectors from the region to confirm this finding.

\section{Acknowledgement}

The authors are thankful to Dr S J Pande, DMO, and Mr Mather Boina, ADMO, Gadchiroli district for their kind support during the study. We would like to thank the laboratory and field staff of ICMR-NIMR Delhi for their assistance in the study. Proper guidance and financial support provided by the Director, National Institute of Malaria Research, Delhi is highly appreciated.

\section{Financial Support}

Intramural funds of ICMR-NIMR, Delhi.

\section{Conflict of Interest: None}

\section{References}

1. World Health Organization [Internet]. World Malaria Report 2018; [cited 2020 Nov 20]. Available from: http://apps.who.int/iris/bitstream/hand 
le/10665/275867/9789241565653-eng.pdf?ua=1

2. National Vector Borne Disease Control Programme [Internet]. Malaria situation in India, Delhi; [cited 2020 Nov 20]. Available from: https://nvbdcp.gov.in/ WriteReadData/l892s/78341804791628840488.pdf

3. Kumar A, Valecha N, Jain T, Dash AP. Burden of Malaria in India: retrospective and prospective view. Am J Trop Med Hyg. 2007;77(6 Suppl):69-78. [PubMed]

4. Dhingra N, Joshi RD, Dhillon GP, Lal S. Enhanced Malaria Control Project for World Bank support under National Malaria Eradication Programme (NMEP). J Commun Dis. 1997;29(3):201-8. [PubMed] [Google Scholar]

5. Office of District Malaria Office, Gadchiroli, Maharashtra state. https://scholar.google.com/scholar?q=Office+of+District+Malaria+Office+,++Gadchiroli,+Maharshtra+.+

6. Sharma VP. Continuing challenge of malaria in India. Curr Sci. 2012;102(5):678-82. [Google Scholar]

7. Singh RK, Kumar G, Mittal PK. Insecticide susceptibility status of malaria vectors in India: A review. Int J Mosq Res. 2014;1(1):5-9. [Google Scholar]

8. Sharma VP, Dev V. Biology \& control of Anopheles culicifacies Giles 1901. Ind J Med Res. 2015;141(5):52536. [PubMed] [Google Scholar]

9. Sharma VP. Fighting malaria in India. Curr Sci. 1998;75:1127-40. [Google Scholar]

10. Rahman J, Roy ML, Singh K. Development of increased tolerance to DDT in Anopheles culicifacies Giles, in the Panch Mahal district of Bombay state, India. Indian J Malariol. 1959;13(2/3):125-30. [Google Scholar]

11. Rajagopal R. Malathion resistance in Anopheles culicifacies in Gujarat. Indian J Med Res. 1977;66:278. [PubMed] [Google Scholar]

12. Dhingra N, Joshi RD, Dhillan GP, Lal S. Enhanced Malaria Control Project by World Bank support under National Malaria Eradication Program (NMEP). J Commun Dis. 1997; 29:201-208.

13. Government of Maharashtra Public Health Department, Directorate of Health Services, [cited 2020 Nov 1]. Available from: https://arogya. maharashtra.gov.in/Site/Form/DiseaseContent. aspx?CategoryDetailsID=gWj0zP09If8=

14. Singh OP, Raghavendra K, Nanda N, Mittal PK, Subbarao SK. Pyrethroid resistance in An. culicifacies in Surat district, Gujarat, west India. Curr Sci. 2002;82:547-50. [Google Scholar]

15. Office of the Registrar General \& Census Commissioner, India [Internet]. Census of India. 2011. Available from: https://www.censusindia.gov.in (accessed on 26.11.2021)

16. World Health Organization [Internet]. Instructions for determining the susceptibility or resistance of adult mosquito to organo-chlorine organophosphate and carbonate insecticides - Diagnostic test. WHO/ VBC/1981-806.

17. World Health Organization. Manual on practical entomology in malaria part II. Methods and techniques. Geneva: World Health Organization; 1975. 191 p.

18. Wattal BL, Kalra NL. Regionwise pictorial keys to the female Indian Anopheles. Bull Nat Soc Mal Other Mosqu Borne Dis. 1961;9:85-138. [Google Scholar]

19. Das BP, Rajagopal R, Akiyama J. Pictorial key to the species of Indian Anopheline mosquitoes. J Pure Appl Zool. 1990;2:131-62. [Google Scholar]

20. Abbott WS. A method of computing the effectiveness of an insecticide. J Econ Entomol. 1925;18:265-7.

21. WHO. Instructions for the bioassay of insecticidal deposits on wall surfaces. VBC/81.5 (WHO/VBC/81.812). Geneva: World Health Organization; 1981.

22. Singh RK, Dhiman RC, Mittal PK, Das MK. Susceptibility of Malaria Vectors to insecticides in Gumla district, Jharkhand state, India. J Vector Borne Dis. 2010;47(2):116-8. [PubMed] [Google Scholar]

23. Singh RK, Dhiman RC, Das MK. Situation analysis of malaria in Godda district of Jharkhand during malaria epidemic. J Commun Dis. 2011;43(2):135-42. [PubMed] [Google Scholar]

24. Singh RK, Mittal PK, Gourshettiwar MP, Pande SJ, Dhiman RC. Susceptibility of malaria vectors to insecticides in Gadchiroli district (Maharashtra), India. J Vector Borne Dis. 2012;49(1):42-4. [PubMed] [Google Scholar]

25. Dhiman RC, Shahi B, Sharma SN, Nanda N, Khargiwarkar VN, Subbarao SK. Persistence of malaria transmission in a tribal area in Maharashtra, India. Curr Sci. 2005;88(3):475-8. [Google Scholar]

26. Rao TR, Bhatia SC. A note on the degree of susceptibility of Anopheles culicifacies to DDT in some parts of Bombay state. Indian J Malariol. 1957;11(3):261-9. [PubMed] [Google Scholar]

27. Deobhankar RB, Palkar ND. Magnitude of DDT resistance in Anopheles culicifacies in Maharashtra state. J Commun Dis. 1990;22:77. [PubMed] [Google Scholar]

28. Krishnamurthy BS, Singh NN. DDT resistance in Anopheles culicifacies Giles, 1901 and Anopheles annularis Van der Wulp 1884 in a village of Meerut district, U.P. Indian J Malariol. 1962;16:375-7. [Google Scholar]

29. Vittal M, Deshpande LB. Development of Malathion resistance in a DDT, $\mathrm{HCH}$ resistant Anopheles culicifacies population in Thane district (Maharashtra). J Commun Dis. 1983;15:144-5. [PubMed] [Google Scholar]

30. Sharma MID, Samnotra KG. A note on gamma BHC and dieldren resistance in Anopheles culicifacies Giles in adjoining areas of Gujarat and Maharashtra states. Bull Natl Soc India Malar Mosq Dis. 1962;10:151-7. 
31. Singh RK, Dhiman RC, Kumar G, Sinha ATS, Dua VK. Susceptibility status of malaria vectors to insecticides in Koderma, Jharkhand. J Commun Dis. 2011;43:273-6. [PubMed] [Google Scholar]

32. Sharma SK, Upadhyay AK, Haque MA, Singh OP, Adak T, Subbarao SK. Insecticide susceptibility status of malaria vectors in some hyper-endemic tribal districts of Orissa. Curr Sci. 2004;87(12):1722-6.

33. Sahu SS, Gunasekaran K, Vijayakumar T, Jambulingam P. Triple insecticide resistance in Anopheles culicifacies: a practical impediment for malaria control in Odisha State, India. Indian J Med Res. 2015 Dec;142(Suppl 1):S59-63. [PubMed] [Google Scholar]

34. Raghavendra K, Vasantha K, Subbarao SK, Pillai MK, Sharma VP. Resistance in Anopheles culicifacies sibling species B and $C$ to Malathion in Andhra Pradesh and Gujarat states, India. J Am Mosq Control Assoc. 1991;7(2):255-9. [PubMed] [Google Scholar]

35. Mishra AK, Chand SK, Barik TK, Dua VK, Raghavendra $\mathrm{K}$. Insecticide resistance status in Anopheles culicifacies in Madhya Pradesh, central India. J Vector Borne Dis. 2012;49(1):39-41. [PubMed] [Google Scholar]

36. Bhatt RM, Sharma SN, Barik TK, Raghavendra K. Status of insecticide resistance in malaria vector, Anopheles culicifacies in Chhattisgarh state, India. J Vector Borne Dis. 2012;49(1):36-8. [PubMed] [Google Scholar]

37. Chand G, Behera P, Bang A, Singh N. Status of insecticide resistance in An. culicifacies in Gadchiroli (Maharashtra) India. Pathog Glob Health. 2017;111(7):362-6. [PubMed] [Google Scholar]

38. Singh N, Khare KK. Forest malaria in Madhya Pradesh: changing scenario of disease and its vectors. Indian J Parasit Dis. 1999;23:105-12.

39. Sahu SS, Patra KP. A study on insecticides resistance in Anopheles fluviatilis and An. culicifacies to $\mathrm{HCH}$ and DDT in the Malkangiri district of Orissa. Indian J Malariol. 1995;32(3):112-8. [PubMed] [Google Scholar]

40. Koekemoer LL, Spillings BL, Christian RN, Lo TC, Kaiser ML, Norton RA, Oliver SV, Choi KS, Brooke BD, Hunt $\mathrm{RH}$, Coetzee M. Multiple insecticide resistance in Anopheles gambiae (Diptera: Culicidae) from Pointe Noire, Republic of the Congo. Vector Borne Zoonotic Dis. 2011;11:1193-200. [PubMed] [Google Scholar]

41. Dabiré KR, Diabaté $A$, Djogbenou $L$, Ouari $A, N^{\prime} G$ uessan R, Ouedraogo JB, Hougard JM, Chandre F, Baldet T. Dynamics of multiple insecticide resistance in the malaria vector Anopheles gambiae in a rice growing area in South-Western Burkina Faso. Malar J. 2008;7(1):188. [PubMed] [Google Scholar]

42. Djouaka RJ, Atoyebi SM, Tchigossou GM, Riveron JM, Irving $\mathrm{H}$, Akoton $\mathrm{R}$, Kusimo MO, Bakare AA, Wondji $\mathrm{CS}$. Evidence of a multiple insecticide resistance in the malaria vector Anopheles funestus in South West
Nigeria. Malar J. 2016;15(1):565. [PubMed] [Google Scholar]

43. Nardini L, Christian RN, Coetzer N, Koekemoer LL. DDT and pyrethroid resistance in Anopheles arabiensis from South Africa. Parasit Vectors. 2013;6(1):229. [PubMed] [Google Scholar]

44. Toé KH, N'Falé S, Dabiré RK, Ranson H, Jones CM. The recent escalation in strength of pyrethroid resistance in Anopheles coluzzi in West Africa is linked to increased expression of multiple gene families. BMC Genomics. 2015;16:146. [PubMed] [Google Scholar]

45. Gunasekaran K, Sahu SS, Jambulingam P, Das PK. DDT Indoor Residual spray, still an effective tool to control Anopheles fluviatilis-transmitted Plasmodium falciparum malaria in India. Trop Med Int Health. 2005;10:160-8. [PubMed] [Google Scholar]

46. Shukla RP, Sharma SN, Bhat SK. Malaria outbreak in Bhojpur PHC of District Moradabad, Uttar Pradesh, India. J Commun Dis. 2002;34:118-23. [PubMed] [Google Scholar]

47. Vittal M, Bhate MR. Bioassay tests on the effectiveness of malathion spraying on Anopheles culicifacies resting on different wall surfaces in Aurangabad town, Maharashtra. Indian J Malariol. 1981;18:124-5. 